\title{
Ischemic Conditioning Is Safe and Effective for Octo- and Nonagenarians in Stroke Prevention and Treatment
}

\author{
Ran Meng ${ }^{1}$ - Yuchuan Ding ${ }^{2} \cdot$ Karam Asmaro $^{2} \cdot$ David Brogan $^{2} \cdot$ Lu Meng $^{3}$ • \\ Meng Sui ${ }^{4} \cdot$ Jingfei Shi $^{1}$ - Yunxia Duan ${ }^{1} \cdot$ Zhishan Sun $^{1} \cdot$ Yang Yu ${ }^{1,5} \cdot$ Jianping Jia $^{1}$. \\ Xunming $\mathbf{J i}^{1}$
}

Published online: 9 May 2015

(C) The American Society for Experimental NeuroTherapeutics, Inc. 2015

\begin{abstract}
Symptomatic intracranial arterial stenosis (SIAS) is very common in octo- and nonagenarians, especially in the Chinese population, and is likely the most common cause of stroke recurrence worldwide. Clinical trials demonstrate that endovascular treatment, such as stenting, may not be suitable for octogenarians with systemic diseases. Hence, less invasive methods for the octogenarian patients are urgently needed. Our previous study (unique identifier: NCT01321749) showed that repetitive bilateral arm ischemic preconditioning (BAIPC) reduced the incidence of stroke recurrence by improving cerebral perfusion (confirmed by single photon emission computed tomography and transcranial Doppler sonography) in patients younger than 80 years of age; however, the safety and effectiveness of BAIPC on stroke prevention in octo- and nonagenarians with SIAS are still unclear. The
\end{abstract}

Ran Meng

victor65@126.com

Xunming Ji

jixunming@vip.163.com

1 Neurology Department and Cerebral Vascular Diseases Research Institute (China-America Institute of Neuroscience), Xuanwu Hospital, Capital Medical University and the Center of Stroke, Beijing Institute for Brain Disorders, Beijing 100053, China

2 Department of Neurosurgery, Wayne State University School of Medicine, Detroit, MI 48201, USA

3 Department of Biochemistry and Molecular Biology, Complex Carbohydrate Research Center, University of Georgia, Athens, GA 30602, USA

4 USC Viterbi School of Engineering, University of Southern California, Los Angeles, CA 90089, USA

5 The 9th Medical College of Peking University, 100038 Beijing, China objective of this study was to evaluate the safety and effectiveness of BAIPC in reducing stroke recurrence in octo- and nonagenarian patients with SIAS. Fifty-eight patients with SIAS were enrolled in this randomized controlled prospective study for 180 consecutive days. All patients enrolled in the study received standard medical management. Patients in the BAIPC group $(n=30)$ underwent 5 cycles consisting of bilateral arm ischemia followed by reperfusion for 5 min each twice daily. Those in the control group $(n=28)$ underwent sham-BAIPC twice daily. Blood pressure, heart rate, local skin status, plasma myoglobin, and plasma levels of thrombotic and inflammatory markers were documented in both groups before beginning the study and for the first 30 days. Finally, the incidences of stroke recurrence and magnetic resonance imaging during the 180 days of treatment were compared. Compared with the control, BAIPC had no adverse effects on blood pressure, heart rate, local skin integrity, or plasma myoglobin, and did not induce cerebral hemorrhage in the studied cohort. BAIPC reduced plasma high sensitive C-reactive protein, interleukin-6, plasminogen activator inhibitor-1, leukocyte count, and platelet aggregation rate and elevated plasma tissue plasminogen activator (all $p<0.01$ ). In 180 days, 2 infarctions and 7 transient ischemic attacks were observed in the BAIPC group compared with 8 infarctions and 11 transient ischemic attacks in the sham BAIPC group $(p<0.05)$. BAIPC may safely inhibit stroke recurrence, protect against brain ischemia, and ameliorate plasma biomarkers of inflammation and coagulation in octo- and nonagenarians with SIAS. A multicenter trial is ongoing.

Clinical Trial Registration: www.clinicaltrials.gov, unique identifier: NCT01570231.

Key Words Remote ischemic preconditioning · octogenarian $\cdot$ nonagenarians $\cdot$ intracranial arterial stenosis . stroke $\cdot$ recurrence 


\section{Introduction}

Symptomatic intracranial atherosclerotic stenosis (IAS) is one of the leading causes of ischemic stroke in China [1], and is associated with a high risk of stroke recurrence secondary to artery-to-artery embolism, hemodynamic failure, or occlusion of the involved vasculature $[1,2]$. The risk of stroke recurrence depends on the severity of the IAS. Patients with $\geq 70 \%$ luminal narrowing have a $35 \%$ risk of stroke recurrence in the first year; this risk decreases to $18 \%$ with antithrombotic therapy [3]. Current therapy available to patients with symptomatic IAS includes medication with or without endovascular intervention [4]. However, recent findings from the SAMMPRIS (Stenting and Aggressive Medical Management for Preventing Recurrent Stroke in Intracranial Stenosis) clinical trial showed a higher risk of early stroke recurrence after stenting compared with aggressive medical management (14.7\% vs $5.8 \%$ ) [5]. Although effective, aggressive medical management does not completely prevent stroke recurrence [6]. In the WASID (Warfarin-Aspirin Symptomatic Intracranial Disease) trial, Kasner et al. [6] reported that $19 \%$ of patients presented with recurrent stroke in the same cerebral hemisphere; additionally, most presented within the first year. Furthermore, the efficacy of stenting for symptomatic IAS remains to be fully evaluated, especially in octo- and nonagenarians, as clinical trials demonstrate that octogenarians may have a greater number of periprocedural events than nonoctogenarians. One study utilizing follow-up angiography suggested that, with $\geq 50 \%$ stenosis, octogenarians assume a 3 -fold higher risk of periprocedural death and/or stroke recurrence compared with non-octogenarians [7]. Hence, more suitable, less invasive treatment methods for the octogenarian patient population are urgently needed.

In the early 1990s, Przyklenk et al. [8] proposed the concept of remote ischemic preconditioning. In their study, brief ischemia of the remote circumflex artery provided protection to ischemic myocardium from subsequent sustained left anterior descending artery occlusion. Emerging clinical trials have corroborated the previous research, suggesting that remote preconditioning may indeed protect the heart [9-11]. In addition, recent findings in experimental animal models and clinical studies suggest that remote ischemic preconditioning may also be beneficial to the brain without exacerbating intracerebral hemorrhage [12-19].

Our previous study (unique identifier: NCT01321749) showed that repetitive bilateral arm ischemic preconditioning (BAIPC) reduced the incidence of stroke recurrence by improving cerebral perfusion (confirmed by single photon emission computed tomography and transcranial Doppler sonography) [18]. We demonstrated a significant reduction in the incidence of recurrent strokes at both 90 days $(5 \%$ vs $23.3 \%)$ and 300 days $(7.9 \%$ vs $26.7 \%$ ) in the BAIPC group when compared with the control. However, our cohort was limited to a patient population younger than 81 years of age. In this study, we test the safety and efficacy of BAIPC in octo- and nonagenarians with symptomatic IAS.

\section{Materials and Methods}

\section{Patient Selection and Study Design}

Patients that presented within 7 days of an ischemic stroke or transient ischemic attack (TIA) were enrolled and randomized if their neurologic symptoms and signs corresponded with the territory of the diseased vessel shown by magnetic resonance imaging (MRI), diffusion weighted imaging (DWI), magnetic resonance angiography (MRA), or computed tomographic angiography (CTA) [19, 20].

The criterion for IAS is similar to that of carotid artery stenosis, and is detailed as intracranial arterial stenosis of at least $70 \%$ by MRA or CTA [21]. The degree of arterial stenosis was based on the MRA or CTA imaging [22, 23] and calculated as 1 (the inside diameter of the blood vessel at the point of greatest stenosis/inside diameter of the normal blood vessel at the distal end). Additional inclusion criteria included 1) age 80-95 years; 2) TOAST (Trial of Org 10172 in Acute Stroke Treatment) subtype 1 of stroke (a stroke caused by IAS) [24, 25]; 3) National Institutes of Health Stroke Scale (NIHSS) score of 0-15, and modified Rankin Scale (mRS) score of 2-4;4) $\mathrm{ABCD}^{2}$ score of 6-7 [20]; 5) stable vital signs, and normal hepatic and renal function; 6) no hemorrhagic tendency.

Exclusion criteria included 1) extracranial arterial stenosis or extracranial in combination with intracranial arterial stenosis; 2) recent (within $72 \mathrm{~h}$ ) intra-arterial or intravenous thrombolysis; 3 ) intracranial hemorrhage; 4) any soft tissue, orthopedic, or vascular injury, and any disease of the extremities that may contraindicate remote preconditioning; 5) acute myocardial infarction; 6) systolic blood pressure $>$ $200 \mathrm{mmHg}$ with medication; 7) hematologic disease; 8) severe hepatic or renal dysfunction; 9) mRS score of $0-1$; 10) severe or unstable concomitant diseases; 11) no informed consent; 12) failure to complete the entire treatment regimen.

The study design is summarized in Fig. 1.

\section{Approvals and Patient Consent}

All study protocols were approved by the local research ethics committee at the Capital Medical University. All participants (or their guardians) signed informed consent prior to enrollment. 


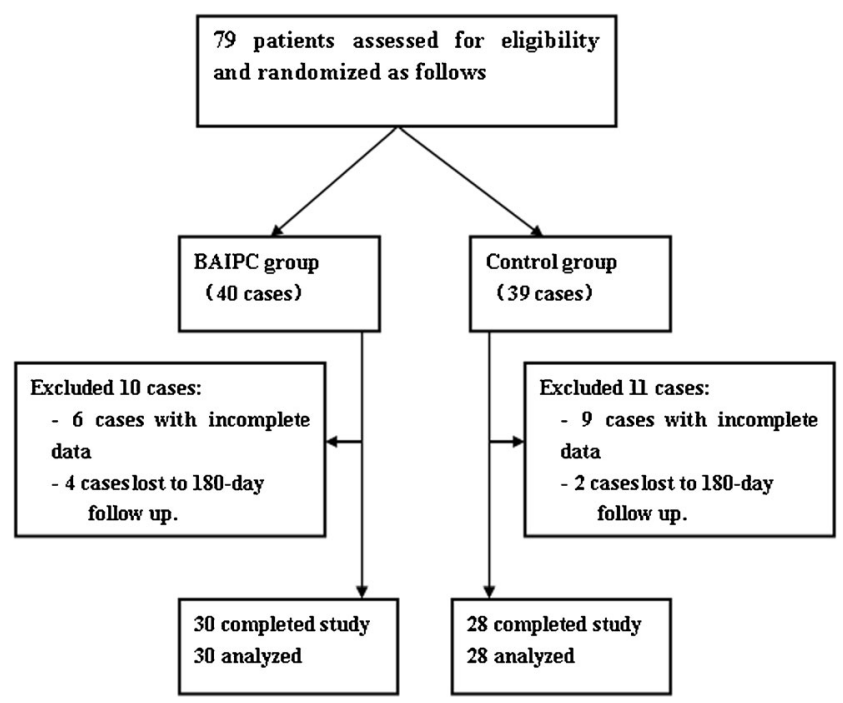

Fig. 1 Schematic diagram of the clinical trial profile. BAIPC=bilateral arm ischemic preconditioning

\section{Registrations}

This study was registered with the Clinical Trial Registration (http://www.clinicaltrials.gov) in 2012 (unique identifier: NCT01570231). The study was conducted from 2008 to 2010 at Xuanwu Hospital.

\section{Procedures}

All patients received standard medical care (including either clopidogrel $75 \mathrm{mg}$ /day in combination with atorvastatin $20 \mathrm{mg}$ /day or aspirin $100 \mathrm{mg}$ /day and clopidogrel $75 \mathrm{mg} /$ day in combination with atorvastatin $20 \mathrm{mg}$ /day). In addition, patients in the BAIPC group $(n=30)$ underwent BAIPC treatment twice daily for 180 consecutive days. The BAIPC procedures were initiated after excluding the presence of unstable plaques and intravascular thrombosis in the upper extremities using a vascular ultrasound. The BAIPC treatment consisted of 5 cycles of simultaneous bilateral upper arm ischemia for $5 \mathrm{~min}$ followed by reperfusion for another $5 \mathrm{~min}$, performed twice daily for 180 consecutive days. The BAIPC procedure was performed using an electric autocontrol device with cuffs that inflated to a pressure of $200 \mathrm{mmHg}$ during the ischemic period (patent number ZL200820123637.X, China) [18, 26]. At the end of the BAIPC treatment, all devices were donated to the patients enrolled. The process was performed with the help of a nurse in the hospital or a family member at home. The device recorded and documented each BAIPC cycle, as well as the patient's heart rate and blood pressure in real time. The BAIPC process could also be stopped at any time if the patient experienced discomfort. Control participants $(n=28)$ underwent a sham BAIPC procedure during which bilateral upper arm cuffs were inflated to a pressure of $30 \mathrm{mmHg}$ for $5 \mathrm{~min}$, followed by $5 \mathrm{~min}$ of relaxation of the cuffs, for a total of 5 cycles twice daily for 180 consecutive days [18, 26]. Aside from the preset cuff pressure during ischemia, the BAIPC and sham BAIPC devices were similar in both appearance and function. The heart rate and blood pressure of each patient before and after BAIPC or sham BAIPC were compared daily for the first 30 days of treatment.

Blood samples were collected before the treatment began (day 0 ) to establish a baseline, and $1 \mathrm{~h}$ after the second BAIPC or sham BAIPC treatment on days 1,15 , and 30 . The samples were analyzed using the immunoturbidimetry assay in a coagulation autoanalyzer (ACL2000; Instrumentation Laboratory, Milan, Italy). Those who examined the samples were blinded to the sample group. Plasma levels of highly sensitive C-reactive protein (hsCRP), leukocyte count, fibrinogen, D-dimer, plasminogen activator inhibitor (PAI)-1, tissue plasminogen activator (TPA), and platelet aggregation rates were assayed and compared between both groups.

\section{Randomization and Masking}

We used a computer-generated blocked randomization technique; the allocation sequence was computer generated and subsequently concealed in consecutively numbered, opaque, sealed envelopes. The trial coordinator randomized the participants to treatment groups after assessment; a total of 58 octoand nonagenarians with symptomatic IAS were randomized into 2 groups in a 1:1 ratio.

While there was a profound difference between the pressure point used in the BAIPC group and that used in control group (200 vs $30 \mathrm{mmHg}$ ), patients were not made aware of either the exact values or what it took to obtain an optimal ischemic event. However, owing to the nature of ischemic preconditioning, it was not possible to completely blind the patients by providing the same pressures between both groups. The research assistants responsible for local skin status evaluations, blood pressure measurements, heart rate measurements, plasma sample assays, and neuroradiology interpretation were blinded to the treatment groups.

\section{Patient Compliance Assurance}

All patients who underwent BAIPC and sham BAIPC treatment demonstrated compliance during their hospital stay. Compliance following hospital discharge was confirmed by the automated real-time recording of the BAIPC device. Patients were required to show the recorded list (print out from the device) at the time of follow-up.

\section{Safety Monitoring End Points}

The following were defined as primary safety end points [26]: 1) Objective signs of tissue or neurovascular injury resulting from BAIPC treatment-inspection by observers blinded to 
the study protocol included palpation of distal radial pulses, visual inspection for local edema, erythema, and/or skin lesions, and palpation for tenderness; 2) development of symptomatic or asymptomatic upper extremity deep vein thrombosis as detected by a vascular ultrasound; 3) elevation of plasma myoglobin levels beyond normal limits after BAIPC treatment; 4) symptomatic hemorrhagic transformation after cerebral infarction; 5) patients not tolerating BAIPC treatment. Any suspicious BAIPC or sham BAIPC treatment related events were to be reported to the investigators by the observers who were blinded to the study protocol.

\section{Clinical Outcome Evaluation End Points}

Stroke and TIA recurrence during the 180 consecutive days of treatment were defined as primary outcome end points. Stroke recurrence was defined as sudden functional deterioration in neurologic status with a decrease of 4 or more in the NIHSS, or a new stroke lesion on MRI/DWI located at the territory of the affected intracranial arteries [27]. Local symptoms and signs corresponding with the territory of the affected intracranial arteries that reappeared and lasted $<24 \mathrm{~h}$ with negative MRI/DWI results were identified as TIA recurrence after the circumstances of blood pressure variations, medication changes, other comorbidities, and depression were excluded. All patients underwent MRI upon enrollment, and at 30 and 180 days of treatment, or at the onset of any new neurologic deficit. The rate of stroke recurrence was reported to the study investigators by observers who were blinded to the study protocol. Secondary outcome end points included improvement of the NIHSS score ( $\geq 8$ points or $\leq 1$ point from the baseline) or a mRS of $0-2$ [28-30].

\section{Blood Evaluation End Points}

Dynamic changes in plasma levels of hsCRP, leukocyte count, fibrinogen, D-dimer, PAI-1 TPA, and platelet aggregation rates in 30 days of BAIPC or sham BAIPC were the blood evaluation end points.

\section{Statistics}

Sample size was calculated with SAS (SAS Institute, Cary, $\mathrm{NC}$, USA), according to the rule of clinical trail $(\alpha=0.05$, $\beta=0.2, \mathrm{P} 0=18 \%$ [5], $\mathrm{P} 1=7.9 \%$ [18], 2 sides, confidence $95 \%$ ), the case number in this Phase IIa study required 30 cases in each group ( 30 cases in Phase I, 30 cases in Phase IIa, 60-70 cases in Phase IIb; 300 cases in Phase III). Descriptive statistics were calculated for all assay data. All values in the text and tables are presented as a mean $\pm \mathrm{SD}$, and were analyzed with a Student's $t$ test. Discrete data were given as counts and percentages, and were compared with $\chi^{2}$ statistics. Average plasma levels of myoglobin at baseline and 1, 15, and
30 days of BAIPC treatment were analyzed with 1-way analysis of variance (ANOVA). Fluctuations of blood pressure and heart rate during the initial 30 days, and the incidence of recurrent stroke, the NIHSS scores, and the mRS scores through the 180 days of BAIPC treatment were displayed on KaplanMeier curves and analyzed with the log-rank test. The effects of smoking, hypertension, and hyperlipidemia on stroke and TIA recurrence were analyzed with regression analysis. For all tests, $p$-values $<0.05$ were considered to be statistically significant. All calculations were performed with the SPSS 13.0 (IBM, Armonk, NY, USA).

\section{Results}

The results presented here are from the Phase IIa study; the Phase IIb study is ongoing.

\section{Patient Characteristics}

Thirty-eight men and 20 women were enrolled in the study (age range 81-95 years). Median age was 83.5 years in the BAIPC group and 84.2 years in the control group. Concomitant risk factors, as well as location and percentage of arterial stenosis, are listed in Table 1. All baseline data were well matched between both groups, including the presence or absence of diabetes, hypertension, and other chronic systemic diseases. Multifocal stenoses were more common than a single vascular stenosis. Medications taken prior to enrollment were well matched between both groups with no statistical difference (Table 1).

Thirty patients in the BAIPC group and 28 patients in the sham BAIPC group finished the whole study with complete data for analysis (Fig. 1). The degree of arterial stenosis was evaluated by MRA and/or CTA; 21 patients in BAIPC group and 18 patients in the sham BAIPC group underwent MRA evaluation only, 7 patients in BAIPC group and 9 patients in the sham BAIPC group underwent CTA evaluation only, and 2 patients in BAIPC group and 1 patient in sham BAIPC group underwent both MRA and CTA evaluations.

\section{Patient Compliance with the Study Protocol}

A total of 58 patients completed 180 days of either BAIPC treatment (30 patients) or sham-BAIPC (28 patients) with proper follow-up. In the BAIPC group, $83.3 \%(25 / 30)$ of patients completed the treatment process without overt discomfort; $16.7 \%(5 / 30)$ complained of mild discomfort but tolerated treatment. Although more than 58 patients enrolled in the study (Fig. 1), some refused to comply with the study parameters (e.g., did not consent to follow-up blood tests) and were therefore not included in the final analysis. 
Table 1 Patient characteristics

\begin{tabular}{|c|c|c|}
\hline Variable & BAIPC $(n=30)$ & Control $(n=28)$ \\
\hline Age $($ mean $\pm \mathrm{SD})$ & $83.5 \pm 2.3$ & $84.2 \pm 1.6$ \\
\hline Sex (male/female) & $18 / 12$ & $17 / 11$ \\
\hline Nationality & 27 Han/3 Hui & 24 Han/4 Hui \\
\hline Time from stroke onset to day of enrollment (day $\pm \mathrm{SD})$ & $4.5+1.5$ & $4.7 \pm 1.2$ \\
\hline Diabetes & $13(43.3)$ & $11(39.2)$ \\
\hline Hypertension & $20(66.7)$ & $18(64.3)$ \\
\hline Smoking & $2(6.7)$ & $3(10.7)$ \\
\hline Hyperlipidemia & $22(73.3)$ & $20(71.4)$ \\
\hline Stenosis+TIA & $10(33.3)$ & $9(32.1)$ \\
\hline Stenosis + stroke & $11(36.7)$ & $10(35.7)$ \\
\hline Stenosis + stroke+TIA & $5(16.7)$ & $6(21.4)$ \\
\hline Intracranial stenosis $(\%)$ & $71.7 \pm 4.8$ & $70.3 \pm 5.9$ \\
\hline Abnormally elevated cholesterol & $23(76.7)$ & $22(78.6)$ \\
\hline Abnormally elevated TRIG & $21(70.0)$ & $20(71.4)$ \\
\hline Abnormally elevated LDL & $26(86.7)$ & $24(85.7)$ \\
\hline Abnormally elevated fibrinogen & $24(80.0)$ & $22(78.6)$ \\
\hline Abnormally elevated D-dimer & $4(13.3)$ & $3(10.7)$ \\
\hline Abnormally elevated hsCRP & $11(36.7)$ & $9(32.1)$ \\
\hline NIHSS (score \pm SD) & $11.3 \pm 2.3$ & $11.1 \pm 2.6$ \\
\hline $\mathrm{mRS}($ score $\pm \mathrm{SD})$ & $3.4 \pm 0.6$ & $3.5 \pm 0.5$ \\
\hline \multicolumn{3}{|l|}{ Locations of stenosis } \\
\hline Right ICA & $6(20.0)$ & $5(17.9)$ \\
\hline Left ICA & $4(13.3)$ & $5(17.9)$ \\
\hline Right MCA & $5(16.7)$ & $5(17.9)$ \\
\hline Left MCA & $5(16.7)$ & $4(14.3)$ \\
\hline BA & $2(6.7)$ & $2(7.1)$ \\
\hline Multifocal stenosis & $8(26.6)$ & $7(25.0)$ \\
\hline ACE inhibitors & $2(6.7)$ & $3(10.7)$ \\
\hline ARBs & $3(10.0)$ & $2(7.1)$ \\
\hline $\mathrm{ARBs}+\mathrm{CCBs}$ & $11(36.7)$ & $10(35.7)$ \\
\hline $\mathrm{CCBs}$ & $4(13.3)$ & $3(10.7)$ \\
\hline Antidiabetic oral therapy & $9(30.0)$ & $8(28.6)$ \\
\hline Antidiabetic insulin therapy & $4(13.3)$ & $3(10.7)$ \\
\hline \multicolumn{3}{|l|}{ Anticoagulation and statin therapy before enrollment } \\
\hline Aspirin & $11(36.7)$ & $12(42.9)$ \\
\hline Clopidogrel & $1(3.3)$ & $2(7.1)$ \\
\hline Aspirin+clopidogrel & 0 & 0 \\
\hline Warfarin & $1(3.3)$ & $1(3.6)$ \\
\hline Statins & $2(6.7)$ & $3(10.7)$ \\
\hline \multicolumn{3}{|l|}{ Anticoagulation and statin therapy after enrollment } \\
\hline Clopidogrel $75 \mathrm{mg} / \mathrm{d}+$ statin $20 \mathrm{mg} / \mathrm{d}$ & $27(90.0)$ & $24(85.7)$ \\
\hline Aspirin $100 \mathrm{mg}+$ clopidogrel $75 \mathrm{mg} / \mathrm{d}+$ statin $20 \mathrm{mg} / \mathrm{d}$ & $3(10)$ & $4(14.3)$ \\
\hline Warfarin & 0 & 0 \\
\hline
\end{tabular}

Values are given as $n(\%)$ unless otherwise indicated. There were no significant differences between the bilateral arm ischemic preconditioning (BAIPC) and control (sham BAIPC) groups (all $p>0.05$ ). TIA =transient ischemic attack; TRIG=trigylcerides; $\mathrm{LDL}=$ low-density lipoprotein; hsCRP=highly sensitive C-reactive protein; NIHS $\mathrm{S}=$ National Institutes of Health Stroke Scale; $\mathrm{mRS}=$ modified Rankin Scale; ICA=internal cervical artery (intracranial section); $\mathrm{MCA}=$ middle cerebral artery; $\mathrm{BA}=$ basilar artery; $\mathrm{ACE}=$ angiotensin-converting enzyme; $\mathrm{ARB}=$ angiotensin receptor blocker; $\mathrm{CCBs}=$ calcium channel blocker 


\section{Safety Monitoring Results}

\section{Blood Pressure and Heart Rate}

To assess the effects of BAIPC on the cardiovascular system, blood pressure and heart rate were measured immediately before and $1 \mathrm{~h}$ after BAIPC and sham BAIPC treatment for the first 30 days of the study. No statistical differences in blood pressure or heart rate were observed between the BAIPC and the control sham BAIPC group before or after treatment (Fig. 2A-D). Furthermore, no statistical difference was observed before or after treatment between the individual groups (BAIPC or the control).

\section{Local Skin and Muscle}

As the BAIPC method involved repeated episodes of brief limb ischemia, potential adverse effects on local skin and muscle integrity were assessed. Transient sporadic petechiaes were observed in 3 cases during the first 30 days of the study, but disappeared soon after without discontinuing BAIPC treatment. No ecchymosis, tenderness to palpation, edema, skin breakage, or other skin lesions were observed. No deep vein thromboses were detected by vascular sonography during or at the end of the 180 days of BAIPC. The average plasma
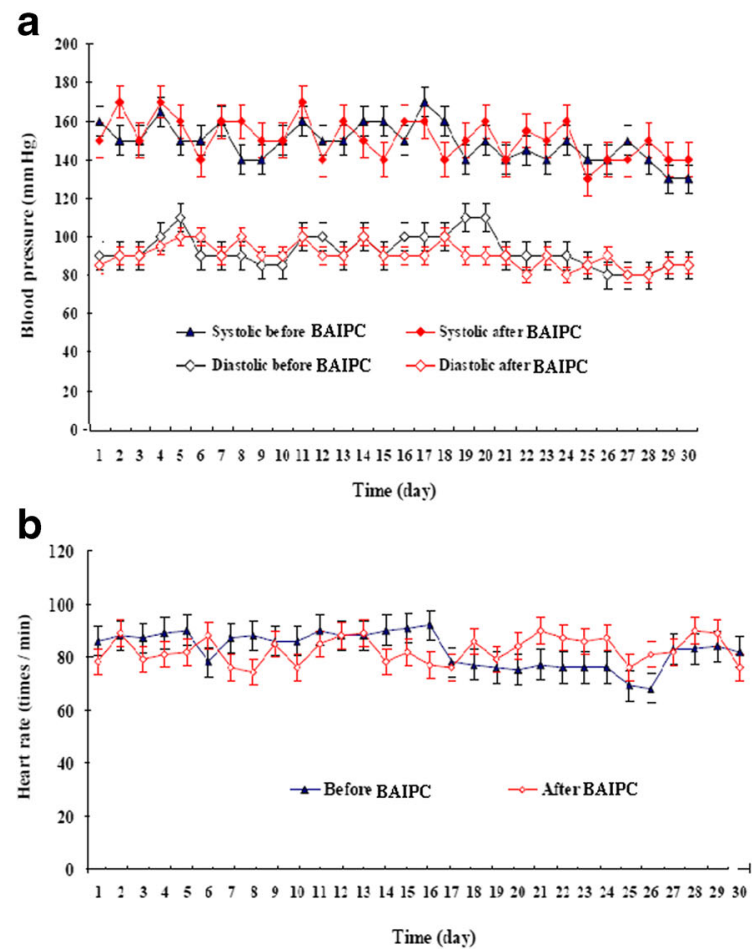

Fig. 2 Cardiovascular parameters during 30 days of bilateral arm ischemic preconditioning (BAIPC) $(n=30)$ and sham BAIPC $(n=28)$ treatment. (A, C) The black curve represents blood pressure immediately before BAIPC/sham BAIPC, and the red curve represents the blood pressure at $1 \mathrm{~h}$ after BAIPC/sham-BAIPC. (B, D) The black curve represents the heart rate immediately before BAIPC/sham BAIPC level of myoglobin was $70.2 \pm 6.5 \mu \mathrm{g} / \mathrm{l}$ at baseline, $69.3 \pm$ $5.5 \mu \mathrm{g} / \mathrm{l}$ on day $1,70.4 \pm 4.1 \mu \mathrm{g} / \mathrm{l}$ on day 15 , and $69.9 \pm$ $5.7 \mu \mathrm{g} / 1$ on day 30 of BAIPC $(F=0.147, p=0.93)$.

\section{Clinical Outcome Evaluation}

\section{Inflammation}

Plasma levels of hsCRP, interleukin (IL)-6, leukocyte count, and platelet aggregation rates were quantified at baseline and at days 1,15 , and 30 following the daily treatment in both BAIPC and control groups (Fig. 3A, B).

Plasma levels of hsCRP and IL-6, as well as platelet aggregation rates, improved significantly at days 15 and 30 in both the BAIPC and sham BAIPC groups when compared with day 0 (1-way ANOVA test, $p<0.01$ ). However, leukocyte count only improved significantly at days 15 and 30 of treatment in the BAIPC group (1-way ANOVA test, $p<0.01)$. No statistically significant improvement in leukocyte count was observed at day 15 or 30 in the sham BAIPC group (1-way ANOVA test, $p>0.05$ ).

Plasma levels of hsCRP were similar in both groups at days 0 and 1 but were significantly lower in the BAIPC group at days 15 and 30 of treatment $[t=7.9$ at day 15 and $t=9.6$ at day

c
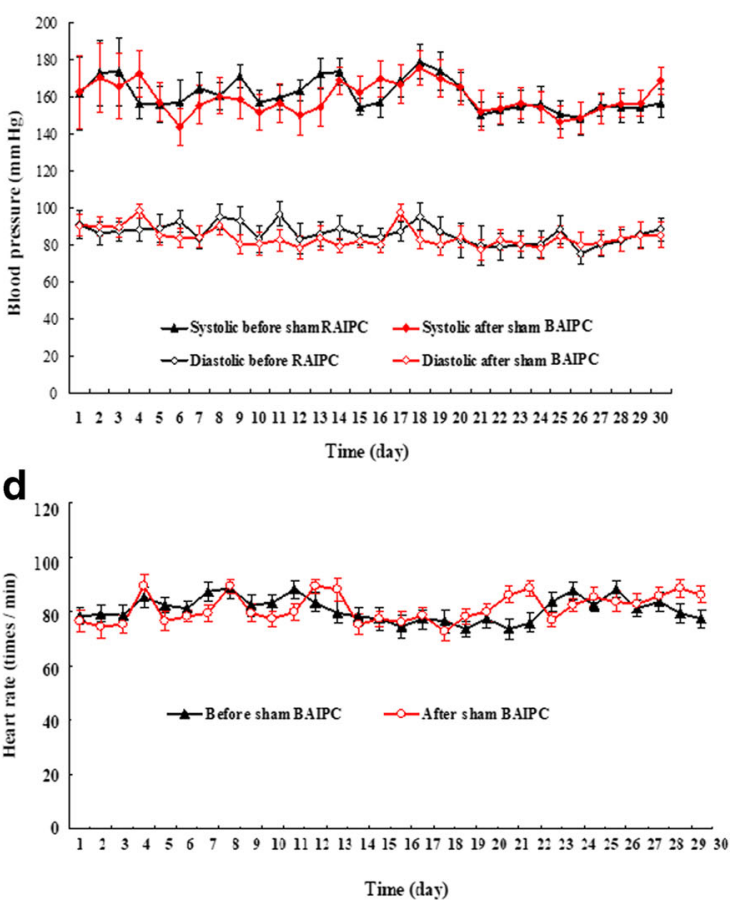

and the red curve represents the heart rate at $1 \mathrm{~h}$ after BAIPC/sham BAIPC. There were no significant effects on the systolic and diastolic blood pressures and heart rates in the BAIPC group (log-rank test: blood pressure and heart rate, before $v s$ after BAIPC, $p=0.063$ and $p=0.191$, respectively; blood pressure and heart rate in the BAIPC $v s$ sham-BAIPC, $p=0.189$ and $p=0.693$, respectively) 

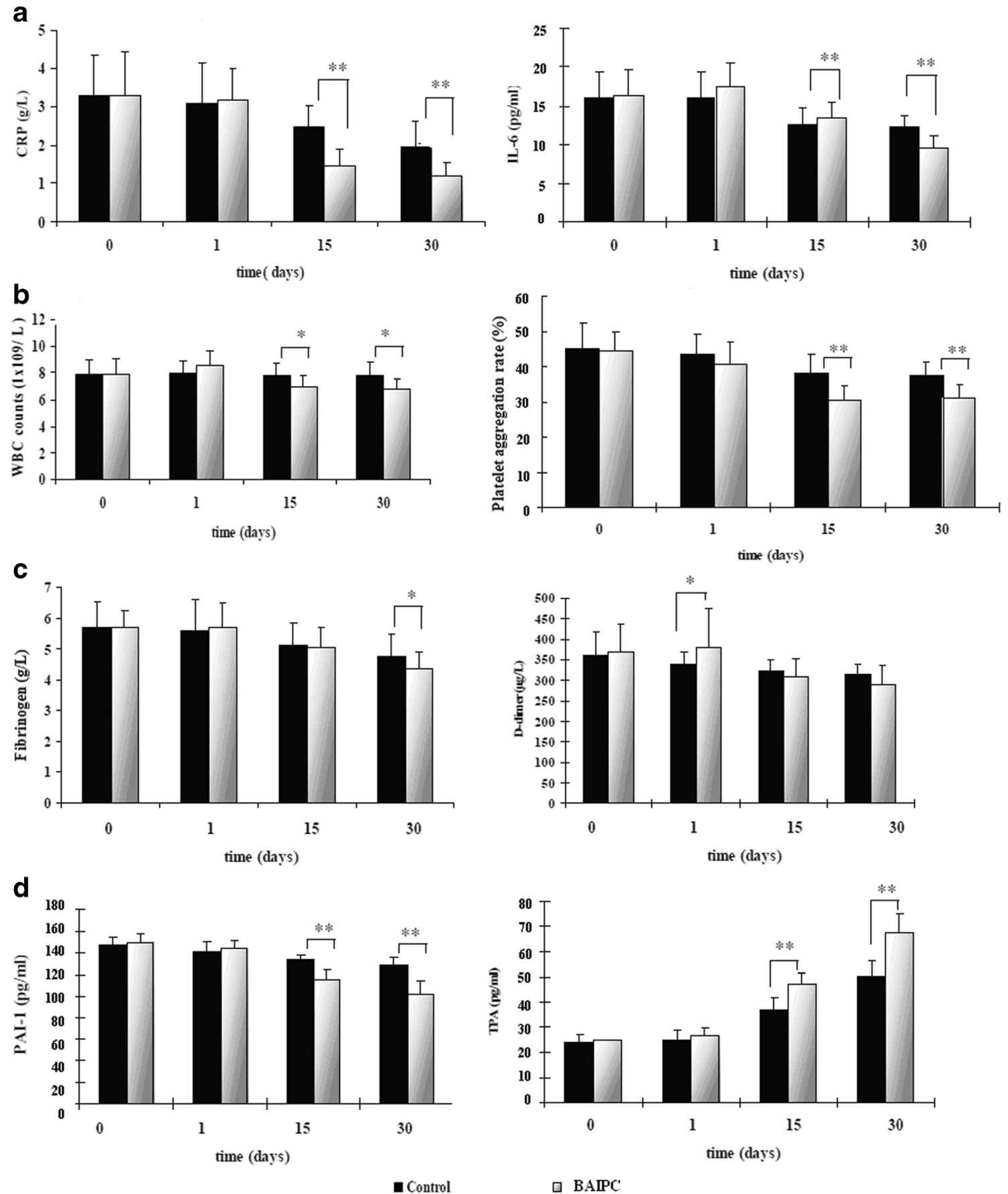

$\square$ BAIPC

Fig. 3 Plasma inflammatory biomarkers. (A) High sensitive C-reactive protein (CRP) and interleukin (IL)-6 levels; (B) leukocyte count and platelet aggregation rates; (C) fibrinogen and D-dimer levels; (D) plasminogen activator inhibitor (PAI)-1 and tissue plasminogen activator (TPA) levels. Blood samples were collected before treatment

and on days 1,15 , and 30 of sham bilateral arm ischemic preconditioning (BAIPC) $(n=28)$ or BAIPC $(n=30)$ treatment $(* p$ $<0.05,{ }^{* *} p<0.01$ ). Black bars represent sham BAIPC (control group); gray bars represent $\mathrm{BAIPC}$. $\mathrm{WBC}=$ white blood cell

30; $p<0.01$ (Fig. 3A)]. Also in the BAIPC group, IL-6 was mildly increased at day 1 but decreased after treatment. By day 30, a significantly lower IL-6 level was observed in the BAIPC group when compared with the control group $(t=6.7$, $p<0.01$; Fig. 3A). The leukocyte count and platelet aggregation rates decrease at days 15 and 30 were more profound in

BAIPC group than in control group (BAIPC $v s$ sham BAIPC: $\mathrm{t}_{15}=3.3, \mathrm{t}_{30}=5.9, \mathrm{t}_{15}=8.3, \mathrm{t}_{30}=6.7$ (all $\left.p<0.01\right)$; Fig. 3B). The results revealed that BAIPC plus standard medical care may further relieve the inflammatory stress of symptomatic IAS when compared with sham-BAIPC plus standard medical care. 


\section{Coagulation and Fibrinolysis}

Plasma levels of fibrinogen, D-dimer, TPA, and PAI-1 were quantified and monitored at days $0,1,15$, and 30 . We observed statistically significant differences in the levels of fibrinogen and PAI- 1 after 15 and 30 days of treatment in the BAIPC group (one-way ANOVA test, $p<0.01$; Fig. 3 C, D). We observed no statistically significant change in the levels of D-dimer in the control group but did observe a mild increase on day 1 in the BAIPC group [ $\mathrm{t}=2.1 ; p<0.05$ (Fig. 3C)]. Increases in the plasma levels of TPA at days 15 and 30 were statistically significant in both groups (one-way ANOVA test, $p<0.01$; Fig. 3D). Fibrinogen levels decreased significantly after 30 days of treatment in the BAIPC group when compared with the control group [ $\mathrm{t}=3.5 ; p=0.002$ (Fig. 3C)]. Also in the BAIPC group, PAI-1 decreased significantly at days 15 and $30[\mathrm{t}=3.6$ and $\mathrm{t}=3.8 ; p<0.01$ (Fig. 3D)]. There was a significant increase in the levels of TPA at days 15 and 30 in the BAIPC group compared with the control group [ $\mathrm{t}=$ 3.0 and $3.2 ; p<0.01$ (Fig. 3D)].

\section{Clinical Outcome}

During the 180 consecutive days of BAIPC or sham BAIPC treatment, the frequency of stroke and TIA recurrent events in both groups, confirmed by MRI/MRA/DWI in combination with clinical symptoms, were compared.

In the BAIPC group 2 recurrent stroke and 7 TIA events occurred (1 patient with 1 stroke only, 1 patient with 1 stroke and 2 TIAs, 1 patient with 2 TIAs, and 1 patient with 3 TIAs). Thus, the percentage of patients with recurrent stroke was $6.7 \%(2 / 30 ; 2$ nonfatal stroke); the percentage of patients with TIAs was $10 \%(3 / 30)$; and the percentage of patients with total recurrent events was $13.3 \%$ $(4 / 30)$.

In the sham BAIPC group 8 recurrent ischemic stroke and 11 TIA events occurred (2 patients with 1 stroke each, 2 patients with 2 strokes each, 1 patient with 2 strokes and 2 TIAs, 3 patients with 1 TIA, 2 patients with 3 TIAs). Thus, the percentage of patients with recurrent stroke was $17.8 \%(5 / 28 ; 4$ nonfatal stroke, 1 fatal stroke); the percentage of patients with TIAs was $21.4 \%(6 / 28)$; and the percentage of patients with total recurrent events was $35.7 \%(10 / 28)$.

The frequency of stroke and TIA recurrence in the BAIPC group was lower than that in sham BAIPC group [log-rank test, $\chi^{2}=8.442 ; p=0.004$ (Fig. 4)]. After adjusting for major confounders associated with stroke and TIA recurrence such as diabetes, smoking, hypertension, hyperlipidemia, and aspirin, clopidogrel, warfarin, and statins use, regression analysis results showed a statistically significant difference between stroke and TIA recurrence in both the groups $(\mathrm{F}=5.52$; $p<0.001$ ).
Fig. 4 Kaplan-Meier curves for primary and secondary end points, logrank test. Bilateral arm ischemic preconditioning (BAIPC) group $(n=30)$ and control group $(n=28)$. (A) Primary end point: stroke and transient ischemic attack (TIA) recurrence. (B) Secondary end point: National Institutes of Health Stroke Scel (NIHSS) score improvement. (C) Secondary end point: modified Rankin Scale (mRS) scores of $0-1$. The green line represents the controls; the blue line represents the BAIPC group. The NIHSS and mRS scores were computed on a near daily basis \pm 1 day. Twenty-six of the 30 patients in the BAIPC group and 25 of the 28 patients in the control group had a brain magnetic resonance at day $30 ; 4$ of the 30 patients in the BAIPC group and 3 of the 28 patients in the control group had brain computed tomography at day 30 ; no symptomatic hemorrhages were observed in either group, indicating that BAIPC does not promote acute cerebral hemorrhage

The NIHSS and mRS scores of patients who experienced recurrent stroke events were included in the analysis. By day 180 , the average NIHSS and mRS scores in the BAIPC group were $2.97 \pm 1.97$ and $1.4 \pm 1.0$, respectively, which were both significantly lower than the control group values of $4.82 \pm 2.72$ and 2.3 \pm 1.1 , respectively $(\mathrm{t}=4.21$ and $\mathrm{t}=3.94$; all $p<0.01$ ). Taken together, these findings suggest that BAIPC may accelerate stroke recovery and improve clinical outcome. KaplanMeier analysis of NIHSS and mRS scores were utilized to track outcomes, and statistically significant improvements were observed in both NIHSS [log-rank test, $\chi^{2}=10.693$; $p<0.01$ (Fig. 4B)] and mRS scores [log-rank test, $\chi^{2}=4.188$; $p=0.04$ (Fig. 4C)].

\section{Discussion}

Symptomatic IAS is a highly comorbid condition that significantly increases one's risk for ischemic stroke [29]. It is responsible for up to $30-50 \%$ of strokes in the Asian population [30]. At present, treatment methods mainly include medication and stenting. However, the placement of a stent is an invasive operation, which is often not suitable for elderly patients with significant comorbidities. Moreover, recent findings from the SAMMPRIS clinical trial reveal that the risk of early recurrent stroke after stenting is higher than that associated with standard medical management alone [5, 31]. However, standard medical management alone does not completely ameliorate the risk of stroke recurrence [5]. Therefore, other treatment methods are urgently needed.

Remote ischemic preconditioning was first demonstrated in the canine myocardium where transient occlusions of a coronary artery resulted in a decrease in myocardial infarct size after permanent occlusion [32]. This phenomenon has now been documented across many different organs and species $[5,11,33-36]$. Our previous study (unique identifier: NCT01321749) showed that BAIPC safely and effectively reduced the incidence of stroke recurrence in symptomatic IAS patients younger than 81 years of age $[14,18]$. However, whether BAIPC was also safe and effective for 
a

Stroke and TIA recurrence

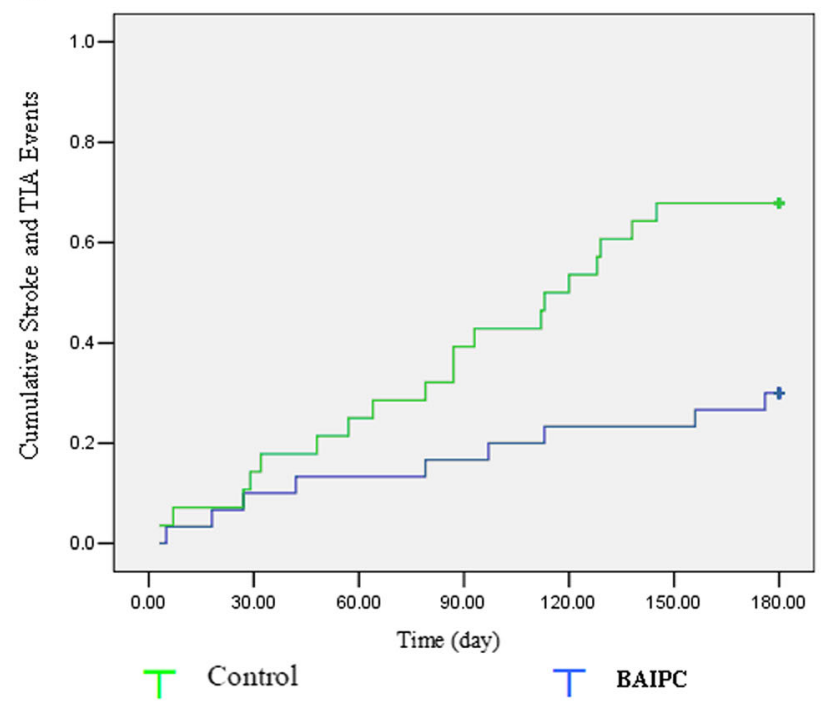

b

The average NIHSS score

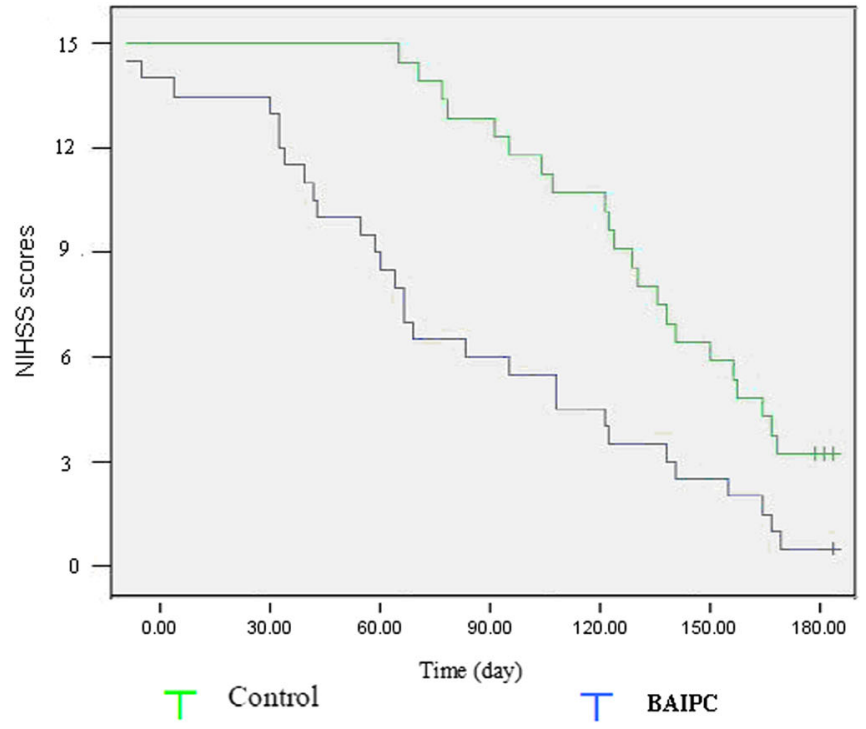

C

The average $\mathrm{mRS}$ score

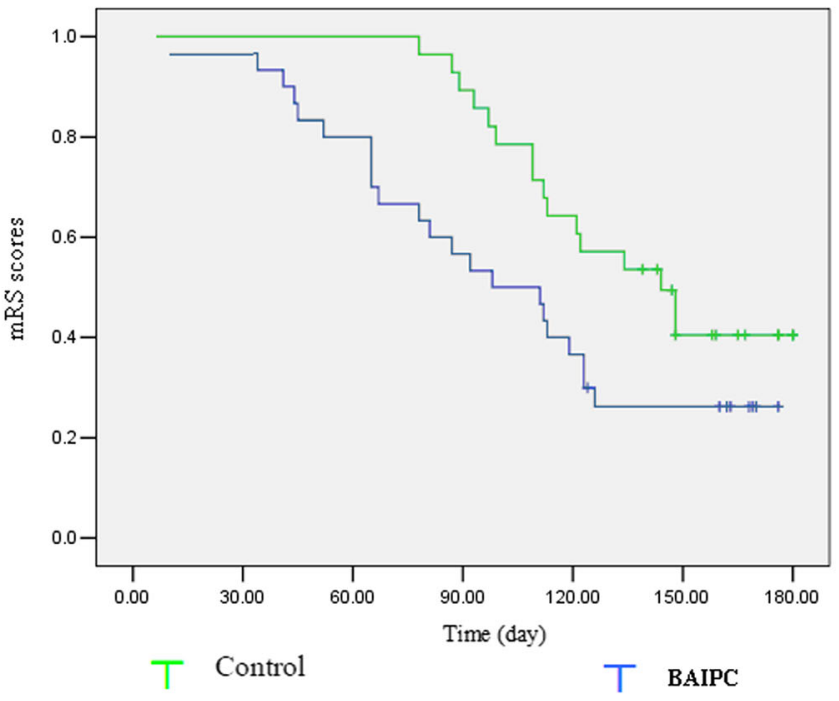


octo- and nonagenarians with symptomatic IAS remained unclear. This study attempted to assess the BAIPC therapeutic approach amongst an older patient population.

Our preliminary findings in octo- and nonagenarians with symptomatic IAS undergoing BAIPC treatment are promising. There were no adverse effects of BAIPC on heart rate, blood pressure, or local tissue during the study. Meanwhile, BAIPC treatment improved clinical outcomes by decreasing the incidences of stroke and TIA recurrence. Our plasma biomarkers results further suggest that the underlying mechanisms of BAIPC may involve the amelioration of inflammatory pathways, increasing endogenous fibrinolytic activity, and reducing endogenous coagulation activity and platelet aggregation.

However, perhaps most promising is that the IAS can be detected by MRA/CTA and transcranial Doppler vascular ultrasound at a common physical examination before stroke onset. Therefore, BAIPC has great potential in clinical application not only to treat, but also to prevent stroke occurrence or recurrence in a cohort of elderly patients with IAS.

Despite the several points mentioned alluding to the safety, efficacy, and potential of BAIPC in octo- and nonagenarians with symptomatic IAS, several points require further study. First, we do not know whether the timing and dosing of our BAIPC therapy are truly optimal. Previous studies suggest that preconditioning yields two protective windows: an acute window that is induced within hours, and a delayed window that may last from 1 to 7 days following treatment $[32,37,38]$. The duration of our BAIPC treatments span a much longer period. Our prolonged approach may be consistent with studies suggesting that long-term (weeks or years) protection may be achieved by repeated doses of preconditioning [11, 39, 40]. Future studies should examine different protocols to find the optimal treatment timing and doses. Another potential limitation involves the exact mechanism of protection. Consistent with data from experimental models, we were able to detect beneficial effects in terms of inflammatory and coagulation biomarkers. However, we recognize that our measurements are only correlational, as our study was not designed to prove causality. Finally, our case numbers are modest in this preliminary study. More powerful studies with larger patient numbers will be required to confirm and validate our BAIPC therapeutic approach fully.

The beneficial effects of BAIPC on the brain have mostly been studied in animal models [34, 41-43]. To our knowledge, this is the first translational study that attempts to apply experimental data to the clinical setting in a cohort of octoand nonagenarians with symptomatic IAS who are not candidates for other therapies. In conclusion, this study suggests that remote ischemic preconditioning may be a simple, noninvasive, and well-tolerated method not only to treat, but also to prevent stroke occurrence and recurrence in elderly patients with symptomatic IAS. Further investigations to develop this promising stroke therapy are ongoing.
Acknowledgments We thank Professor Xiaoying Wang for helping revise the manuscript.

Required Author Forms Disclosure forms provided by the authors are available with the online version of this article.

Conflict of Interest RM, YD, KA, DB, LM, JS, YD, ZS, BW, and JJ report no disclosures or conflict of interest. $\mathrm{XJ}$ is one of the inventors of the electric auto-control device which has been is patented in china (ZL200820123637.X).

Funding Sources This study was sponsored by the National Natural Science Foundation (81371289), China, The Foundation of the Beijing High Level Health Systems (Talented Technical Personnel Program 2009-03-02), China; and The National Key Department of Neurology funded by Chinese Health and Family Planning Committee

\section{References}

1. Wong $\mathrm{KS}, \mathrm{Li} \mathrm{H}$. Long-term mortality and recurrent stroke risk among chinese stroke patients with predominant intracranial atherosclerosis. Stroke 2003;34:2361-2366.

2. Prabhakaran S, Romano JG. Current diagnosis and management of symptomatic intracranial atherosclerotic disease. Curr Opin Neurol 2011;25:18-26.

3. Kasner SE, Chimowitz MI, Lynn MJ, et al. Predictors of ischemic stroke in the territory of a symptomatic intracranial arterial stenosis. Circulation 2006;113:555-563.

4. Turan TN, Derdeyn CP, Fiorella D, et al. Treatment of atherosclerotic intracranial arterial stenosis. Stroke 2009;40:2257-2261.

5. Chimowitz MI, Lynn MJ, Derdeyn CP, et al. Stenting versus aggressive medical therapy for intracranial arterial stenosis. N Engl J Med 2011;365:993-1003.

6. Kasner SE, Lynn MJ, Chimowitz MI, et al. Warfarin vs aspirin for symptomatic intracranial stenosis: Subgroup analyses from WASI D. Neurology 2006;67:1275-1278.

7. Suri MF, Tariq N, Siddiq F, et al. Intracranial angioplasty and/or stent placement in octogenarians is associated with a threefold greater risk of periprocedural stroke or death. J Endovasc Ther 2010;17:314-319.

8. Przyklenk K, Bauer B, Ovize M, et al. Regional ischemic 'preconditioning' protects remote virgin myocardium from subsequent sustained coronary occlusion. Circulation 1993;87:893-899.

9. Hausenloy DJ, Yellon DM. The therapeutic potential of ischemic conditioning: An update. Nat Rev Cardiol 2011;8:619-629.

10. Hoole SP, Heck PM, Sharples L, et al. Cardiac remote ischemic preconditioning in coronary stenting (crisp stent) study: A prospective, randomized control trial. Circulation 2009;119:820-827.

11. Botker HE, Kharbanda R, Schmidt MR, et al. Remote ischaemic conditioning before hospital admission, as a complement to angioplasty, and effect on myocardial salvage in patients with acute myocardial infarction: A randomised trial. Lancet 2010;375:727-734.

12. $\mathrm{Hu} \mathrm{S}$, Dong $\mathrm{H}$, Zhang $\mathrm{H}$, et al. Noninvasive limb remote ischemic preconditioning contributes neuroprotective effects via activation of adenosine a1 receptor and redox status after transient focal cerebral ischemia in rats. Brain Res 2012;1459:81-90.

13. Zare Mehrjerdi F, Aboutaleb N, Habibey R, et al. Increased phosphorylation of mTOR is involved in remote ischemic preconditioning of hippocampus in mice. Brain Res 2013;1526:94-101.

14. Geng X, Ren C, Wang T, et al. Effect of remote ischemic postconditioning on an intracerebral hemorrhage stroke model in rats. Neurol Res 2012;34:143-148. 
15. Hoda MN, Siddiqui S, Herberg S, et al. Remote ischemic perconditioning is effective alone and in combination with intravenous tissue-type plasminogen activator in murine model of embolic stroke. Stroke 2012;43:2794-2799.

16. Jensen HA, Loukogeorgakis S, Yannopoulos F, et al. Remote ischemic preconditioning protects the brain against injury after hypothermic circulatory arrest. Circulation 2012;123:714-721.

17. Hougaard KD, Hjort N, Zeidler D, et al. Remote ischemic preconditioning in thrombolysed stroke patients: Randomized study of activating endogenous neuroprotection - design and MRI measurements. Int J Stroke 2013; 8:141-146.

18. Meng R, Asmaro K, Meng L, et al. Upper limb ischemic preconditioning prevents recurrent stroke in intracranial arterial stenosis. Neurology 2012;79:1853-1861.

19. Leone MA, Gaviani P, Ciccone G. Inter-coder agreement for ICD9-CM coding of stroke. Neurol Sci 2006;27:445-448.

20. Sheehan OC, Merwick A, Kelly LA, et al. Diagnostic usefulness of the $\mathrm{ABCD} 2$ score to distinguish transient ischemic attack and minor ischemic stroke from noncerebrovascular events: The north Dublin TIA study. Stroke 2009;40:3449-3454.

21. Brott TG, Hobson RW 2nd, Howard G, et al. Stenting versus endarterectomy for treatment of carotid-artery stenosis. N Engl J Med 2010;363:11-23

22. Coutts SB, Eliasziw M, Hill MD, et al. An improved scoring system for identifying patients at high early risk of stroke and functional impairment after an acute transient ischemic attack or minor stroke. Int J Stroke 2008;3:3-10.

23. North American Symptomatic Carotid Endarterectomy Trial Collaborators. Beneficial effect of carotid endarterectomy in symptomatic patients with high-grade carotid stenosis. N Engl J Med 1991;325:445-453.

24. Goldstein LB, Jones MR, Matchar DB, et al. Improving the reliability of stroke subgroup classification using the Trial of Org 10172 in Acute Stroke Treatment (TOAST) criteria. Stroke 2001;32:1091-1098.

25. Howard VJ, Lutsep HL, Mackey A, et al. Influence of sex on outcomes of stenting versus endarterectomy: A subgroup analysis of the Carotid Revascularization Endarterectomy versus Stenting Trial (CREST). Lancet Neurol 2011;10:530-537.

26. Koch S, Katsnelson M, Dong C, et al. Remote ischemic limb preconditioning after subarachnoid hemorrhage: A phase Ib study of safety and feasibility. Stroke 2011;42:1387-1391.

27. Wang Y, Zhao X, Liu L, et al. Prevalence and outcomes of symptomatic intracranial large artery stenoses and occlusions in China: the Chinese Intracranial Atherosclerosis (CICAS) Study. Stroke 2014 45:663-669.
28. Shinohara Y, Katayama Y, Uchiyama S, et al. Cilostazol for prevention of secondary stroke (CSPS 2): An aspirin-controlled, double-blind, randomised non-inferiority trial. Lancet Neurol 2010;9: 959-968

29. Gorelick PB, Wong KS, Bae HJ, et al. Large artery intracranial occlusive disease: A large worldwide burden but a relatively neglected frontier. Stroke 2008;39:2396-2399.

30. Wong LK. Global burden of intracranial atherosclerosis. Int $\mathbf{J}$ Stroke 2006;1:158-159.

31. Broderick JP. The challenges of intracranial revascularization for stroke prevention. N Engl J Med 2011;365:1054-1055.

32. Murry CE, Jennings RB, Reimer KA. Preconditioning with ischemia: A delay of lethal cell injury in ischemic myocardium. Circulation 1986;74:1124-1136.

33. Jensen HA, Loukogeorgakis S, Yannopoulos F, et al. Remote ischemic preconditioning protects the brain against injury after hypothermic circulatory arrest. Circulation 2011;123:714-721.

34. Malhotra S, Naggar I, Stewart M, et al. Neurogenic pathway mediated remote preconditioning protects the brain from transient focal ischemic injury. Brain Res 2011;1386:184-190.

35. Kitagawa K, Matsumoto M, Kuwabara K, et al. 'Ischemic tolerance' phenomenon detected in various brain regions. Brain Res 1991;561:203-211.

36. Blanco M, Lizasoain I, Sobrino T, Vivancos J, Castillo J. Ischemic preconditioning : a novel target for neuroprotective therapy. Cerebrovasc Dis 2006;21(Suppl. 2):38-47.

37. Dirnagl U, Becker K, Meisel A. Preconditioning and tolerance against cerebral ischaemia: From experimental strategies to clinical use. Lancet Neurol 2009;8:398-412.

38. Dave KR, Saul I, Prado R, et al. Remote organ ischemic preconditioning protect brain from ischemic damage following asphyxial cardiac arrest. Neurosci Lett 2006;404:170-175.

39. Yannopoulos FS, Makela T, Niemela E, et al. Improved cerebral recovery from hypothermic circulatory arrest after remote ischemic preconditioning. Ann Thorac Surg 2010;90:182-188.

40. Keep RF, Wang MM, Xiang J, et al. Is there a place for cerebral preconditioning in the clinic? Transl Stroke Res 2010;1:4-18.

41. Ren C, Gao X, Steinberg GK, et al. Limb remote-preconditioning protects against focal ischemia in rats and contradicts the dogma of therapeutic time windows for preconditioning. Neuroscience 2008; 151:1099-1103.

42. Hahn CD, Manlhiot C, Schmidt MR, et al. Remote ischemic perconditioning: A novel therapy for acute stroke? Stroke 2011;42: 2960-2962.

43. Sun XC, Xian XH, Li WB, et al. Activation of p38 mapk participates in brain ischemic tolerance induced by limb ischemic preconditioning by up-regulating Hsp 70. Exp Neurol 2010;224:34. 